\title{
Introduction to Socio-Technical Issues in Organizational Information Technologies Minitrack
}

\author{
Michael B. Knight \\ Texas A\&M - Kingsville \\ Michael.Knight@tamuk.edu
}

\author{
Dragos Vieru \\ Distance Learning University of \\ Quebec \\ dragos.vieru@teluq.ca
}

\author{
B. Dawn Medlin \\ Appalachian State University \\ medlinbd@appstate.edu
}

This track appeared at HICSS in the early 2000s and was adopted as a new track due to the issues it addressed at that time and has continued to address as HICSS celebrates its $50^{\text {th }}$ year of service. The topics in this track for presentation are issues related to information technology and social systems, as well as other related social issues that are most aligned with the human factor in terms of information systems planning, development and utilization. The track also aligns itself with the International Journal of Social and Organizational Dynamics in order to provide avenues for research and publishing related to the inherit topics of the conference. Dr. Knight serves as the Editor of the IJSODIT journal and Dr. Vieru and Medlin serve on the board, thus paralleling the two.

Papers in the last several years have addressed issues such as distrust in information systems, technical security measures, diversity in organizational social media adoption, and interpreting information security policies, as well as the inter-organizational dynamics of eco-systems and the establishing of ICT Governance for Regional Information Infrastructures in Healthcare, As you can see the topics offer key topic areas related to (1) trust, (2) relationships, (3) human interaction, (4) diversity and culture in the IT workforce, among other social and IT issues.

For HICSS 50, six (6) papers were accepted. The authors of the first paper titled "An Exploration of Anchoring Female Millennial Students to an IS/IT Career Path: The CLASS Model," contend that addressing the demographic influences on the large generation entering the workforce will provide advantages to research and practice.

The next authors' paper "Strengthening social ties via ICT in the organization," suggest that especially asynchronous and synchronous text based interaction and communication history "forms an effective mechanism for an organization to facilitate social ties.
"The Effect of Virtual Team Membership Change on Social Identity Development: A Case from Higher Education in Norway," was the third accepted paper. The authors asked the question of "What factors may influence the perceptions and development of a group social identity on a new virtual team? Prior experiences with virtual team environments, and other factors were addressed to see their relevance to the topic.

"It's Not Just About Attention to Details: Redefining the Talents Autistic Software Developers Bring to Software Development," offers insights into research related to hiring initiatives targeting individuals with Autism Spectrum Condition (ASC) for software development roles. Additionally, the researchers compare the talents of individuals with ASC to skills required of software developers and identify synergies between the developer skills and strengths of individuals with ASC, such as systemizing, information processing, and specialization of interests

"A Typology for Organizational ICT Practice," offers findings that would suggest a typology for organizational ICT practice which combines seminal literature from Parsons and Bourdieu with more contemporary ideas around sociomateriality. The resulting theoretical contribution of this article provides empirical insights into the underlying factors which need to be investigated in order to gain a holistic understanding of sociomaterial practice.

The final paper titled "Understanding Private Technological Availability and Technological Turbulence as Executives' Job Conditions: A WorkFamily Perspective," offers a study concerning the work-family culture as it relates positively to organizational performance, through executives' satisfaction with work-family balance and executive performance, whereas work-family program availability does not affect executives' satisfaction with work-family balance. 